\title{
Acroqueratosis Paraneoplásica (Síndrome de Bazex)
}

\author{
Lorente Luna M. ${ }^{1}$, Vidal Asensi S. ${ }^{2}$, López Galán C. ${ }^{3}$
}

Sanid. mil. 2015; 71 (4): 247-248; ISSN: 1887-8571

\section{RESUMEN}

La acroqueratosis paraneoplásica o Síndrome de Bazex es una dermatosis infrecuente, habitualmente asociada a neoplasias de los tractos respiratorio superior y gastrointestinal. En muchos casos precede al diagnóstico del tumor subyacente, por lo que su reconocimiento es de suma importancia.

PALABRAS CLAVE: Acroqueratosis paraneoplásica, Sindrome de Bazex, dermatosis psoriasiforme.

\section{Acrokeratosis paraneoplastica (Bazex Syndrome)}

SUMMARY: Acrokeratosis paraneoplastica (Bazex Syndrome) is a rare dermatosis, usually associated with neoplasms of the upper respiratory and gastrointestinal tracts. Often precedes diagnosis of the underlying tumor, so its recognition is mandatory.

KEYWORDS: Acrokeratosis paraneoplastica, Bazex Syndrome, psoriasiform dermatosis.

\section{OBSERVACIÓN CLÍNICA}

Varón de 83 años con antecedente de adenocarcinoma de pulmón T3N0M0 tratado mediante cirugía y radioterapia en 2013 y con posterior recaída en 2014. Consultó por lesiones cutáneas eritematodescamativas bien delimitadas de inicio en dorso de manos (fig.1a)

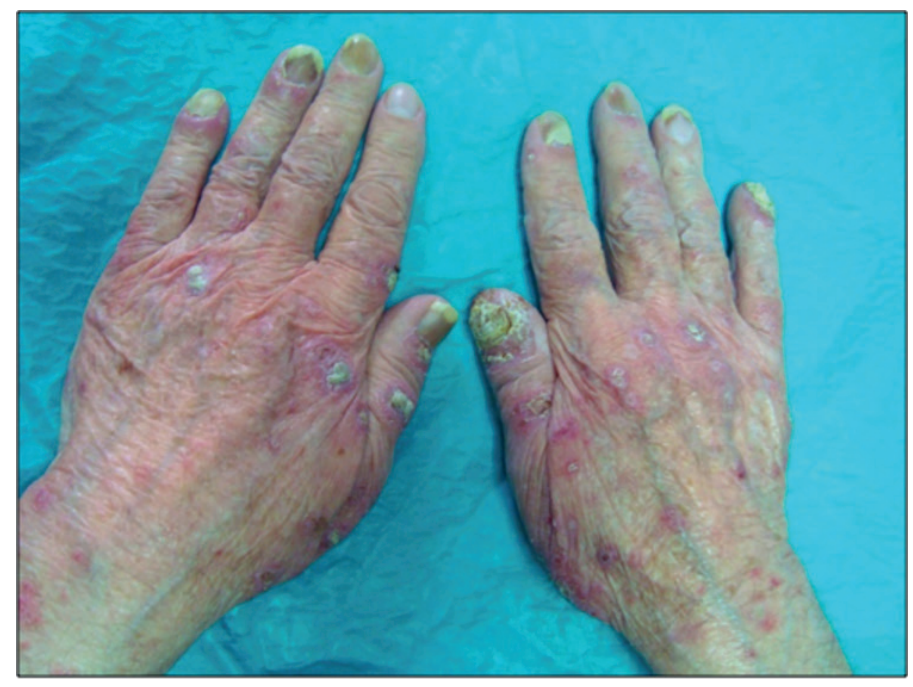

Figura 1a. Imagen clínica. Pápulas y placas eritematosas, con superficie descamativa e hiperqueratósica con distrofia ungueal importante. Nótese la coloración amarillenta deslustrada, la hiperqueratosis y onicolisis distal que afecta a todas las uñas de las manos.

\footnotetext{
FEA. Servicio de Dermatología.

2 Tcol. Médico. Servicio de Dermatología.

${ }^{3}$ Cte. Médico. Servicio de Anatomía Patológica. Hospital Central de la Defensa Gómez Ulla. Madrid. España.
}

Dirección para correspondencia: Dra. Mónica Lorente Luna. Hospital Central de la Defensa Gómez Ulla. Servicio de Dermatología. Edificio de Mínimos, planta 2. Glorieta del Ejercito 1.28047 Madrid.m.lorente.luna@gmail.com; mlorlun@mde.es y pabellones auriculares que se extendieron a tronco (fig. 1b) y extremidades. El estudio histológico reveló hiperplasia psoriasiforme, con paraqueratosis y migración de neutrófilos hacia la epidermis y discreto infiltrado inflamatorio perivascular en dermis papilar (fig.2a, 2 b y 2c), sin identificación de agentes infecciosos compatible con el diagnóstico de acroqueratosis paraneoplásica. Se pautó tratamiento tópico sintomático con corticoide tópico y vaselina salicílica con mejoría parcial de las lesiones.

\section{DISCUSIÓN}

La acroqueratosis paraneoplásica fue descrita por Bazex en $1965^{1}$ y se considera una dermatosis infrecuente, con menos de 170 casos descritos ${ }^{2}$. Se asocia habitualmente a neoplasias de los tractos respiratorio superior y gastrointestinal, aunque también se ha asociado con menor frecuencia a neoplasias pulmonares, de próstata

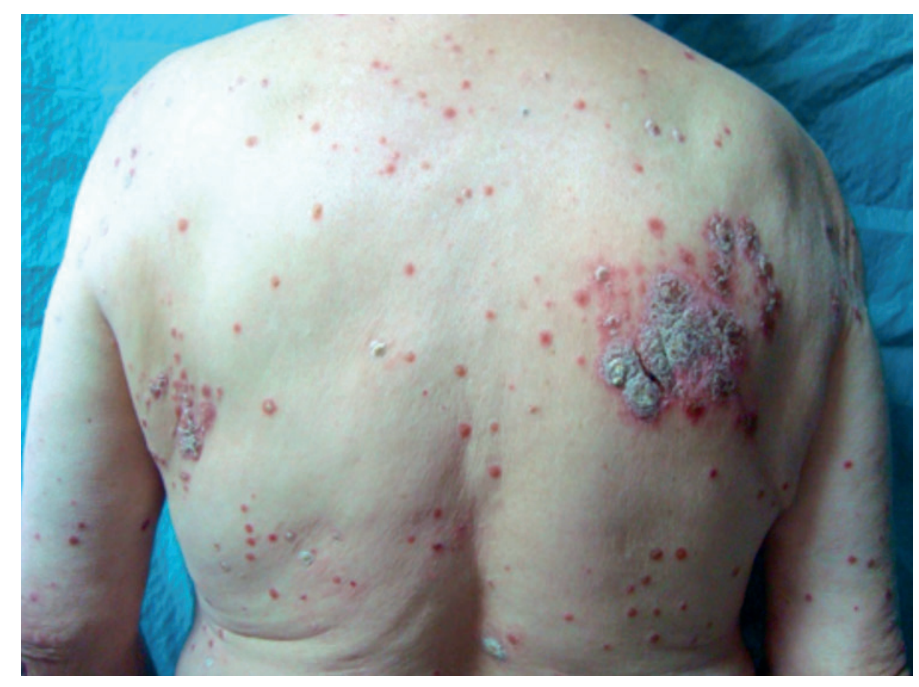

Figura 1b. Imagen clínica. Extensión de las lesiones eritematodescamativas e hiperqueratósicas en espalda. 


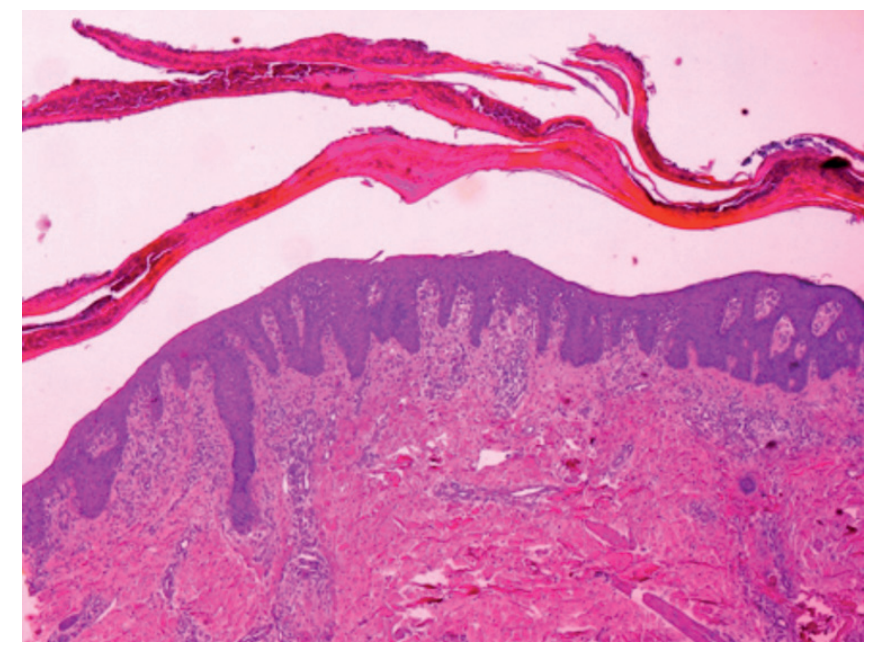

Figura 2a. H-E: panorámica. Hiperplasia psoriasiforme, en la unión dermoepidérmica se aprecia adelgazamiento de la placa suprapapilar. (4x).

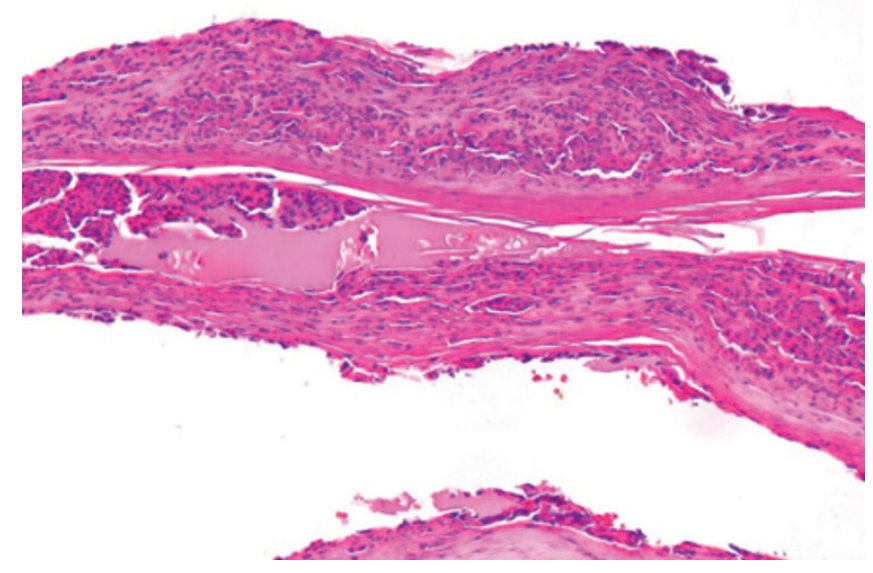

Figura 2b. H-E: detalle del estrato córneo. Paraqueratosis y presencia de neutrófilos. (20x).

y de forma aislada a neoplasias gástricas, tímicas y de médula ósea entre otras ${ }^{3,4}$. Afecta en su mayoría a varones de edad media.

Los síntomas cutáneos preceden al diagnóstico del tumor en más del $60 \% 0^{2-6}$ y se suelen acompañar de adenopatías regionales ${ }^{5}$. Su patogenia continúa sin dilucidarse. Se han postulado como mecanismos la reactividad cruzada entre antígenos del tumor y de la piel que generaría una respuesta de las células $\mathrm{T}$ y también la producción de factores de crecimiento por parte del tumor, que favorecerían la aparición de las lesiones hiperqueratósicas ${ }^{2}$. Se distinguen 3 estadios clínicos $^{1}$; en el estadio 1 aparecen lesiones psoriasiformes en manos y pies, pabellones auriculares y dorso nasal. En el estadio 2 se añade queratodermia palmoplantar y aumento de la extensión. En el estadio 3 las lesiones son generalizadas. Las lesiones cutáneas son habitualmente asintomáticas ${ }^{5}$. En fototipos altos la hiperpigmentación es un hallazgo predominante. La afectación ungueal supera el 75\%, con hiperqueratosis subungueal, estrías longitudinales y coloración amarillenta ${ }^{3,6}$.

El diagnóstico es clínico ya que la histología no es específica. Los hallazgos clásicos incluyen hiperqueratosis con paraqueratosis,

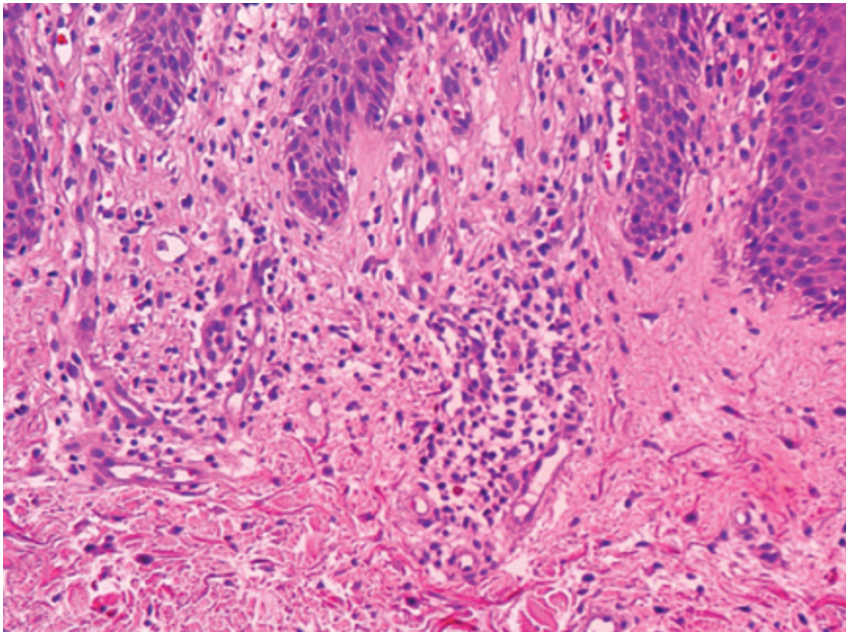

Figura 2c. H-E: detalle de la dermis papilar. Edema intersticial y dilatación de capilares con discreto infiltrado inflamatorio perivascular de predominio mononuclear con aislados neutrófilos. $(40 x)$.

acantosis e infiltrados inflamatorios perivasculares, necrosis de queratinocitos y degeneración vacuolar de la membrana basal, a veces indistinguibles de la psoriasis o el eczema crónico ${ }^{3,5}$.

Se debe hacer diagnóstico diferencial con otras dermatosis eritemato-escamosas, como la psoriasis, el eccema resistente a tratamiento y las micosis superficiales ${ }^{4}$. Si se presenta en pacientes mayores de 60 años se deben considerar también las toxicodermias psoriasiformes y la micosis fungoide ${ }^{3}$. Ante un paciente con sospecha de acroqueratosis paraneoplásica y sin neoplasia previa subyacente, se deben solicitar una radiografía de tórax, analítica sanguínea con bioquímica hepática, renal, perfil férrico y velocidad de sedimentación globular, una exploración otorrinolaringológica completa y sangre oculta en heces. El resto de estudios complementarios deben ir dirigidos en función de la sospecha clínica y los hallazgos obtenidos en las exploraciones previas ${ }^{4}$. Para la resolución de las lesiones es fundamental el tratamiento de la neoplasia subyacente.

El síndrome de Bazex es una entidad habitualmente refractaria a los tratamientos ${ }^{6}$. Como terapias sintomáticas, se han utilizado los corticoides tópicos y sistémicos, el ácido salicílico, los análogos de la vitamina $\mathrm{D}$, los retinoides tópicos y sistémicos y la fototerapia con respuesta variable.

\section{BIBLIOGRAFÍA}

1. Bazex A, Salvador R, Dupré A, Christol B. Syndrome paranéoplasique à type d'hyperkératose des extremités. Guérison après le traitement de l'épithelioma laryngé. Bull Soc Fr Dermatol Syphiligr 1965;72: 182.

2. Tay LK, Pang SM. Late-onset acrokeratosis paraneoplastica of Bazex associated with metastatic adenocarcinoma of the colon. Cutis. 2014;93:E3-5.

3. Bolognia JL. Bazex syndrome: acrokeratosis paraneoplastica. Semin Dermatol 1995; 14: 84-89.

4. Valdivielso M, Longo I, Suárez R, Huerta M, Lázaro P. Acrokeratosis paraneoplastica: Bazex syndrome. J Eur Acad Dermatol Venereol. 2005;19:340-4.

5. Poligone B, Christensen SR, Lazova R, Heald PW. Bazex syndrome (acrokeratosis paraneoplastica). Lancet. 2007 10;369:530.

6. Fleming JD, Stefanato CM, Attard NR. Bazex syndrome (acrokeratosis paraneoplastica). Clin Exp Dermatol. 2014;39:955-6. 